\title{
Non-Linear Transmission Lines for Pulse Shaping in Silicon
}

\author{
Ehsan Afshari and Ali Hajimiri \\ California Institute of Technology (Caltech) \\ Pasadena, CA, USA \\ Caltech MC 136-93, 1200 E. California blvd., Pasadena, CA 91125
}

\begin{abstract}
Non-linear transmission lines (NLTL) are used for pulse shaping. We developed the theory of pulse propagation through the NLTL. The problem of a wide pulse degenerating into multiple pulses rather than a single pulse is solved by using a novel gradually scaled NLTL. We exploit certain favorable properties of accumulation mode MOS varactors to design an NLTL that can sharpen both rising and falling edges, simultaneously. There is a good agreement among the theory, simulations, and measurements.
\end{abstract}

\section{Introduction}

The concept of a solitary wave was introduced to science by John Scott Russell more than a century ago [1]. In 1834 he observed a wave formed when a boat which was rapidly drawn along a narrow channel by a pair of horses suddenly stopped. According to his diary, this wave continued, "at great velocity, assuming the form of a large solitary elevation, a well-defined heap of water that continued its course along the channel apparently without change of form or diminution of speed". These solitary waves, now called 'solitons', have become important items of research in diverse fields of physics and engineering. There is a considerable body of work on solitons in applied mathematics (e.g., [2]), applied physics, especially in optics (e.g. [3] and [4]), and few works in electronics [5]. The ability of solitons to propagate with small dispersion can be used as an effective means to transmit data modulated as short pulses over long distances.

An important related application is pulse sharpening for the more traditional non-return-to-zero (NRZ) data transmission in digital circuits by improving the rise and fall times of pulses. Improving the transitions by shrinking the rise and fall times of pulses can be useful in other applications, such as high-speed sampling and timing systems. Non-linear transmission lines (NLTLs) sharpening either of the rising or the falling edge of a pulse have been demonstrated on a GaAs technology [6]. However, to the best of our knowledge, there has been no demonstration of simultaneous reduction of both rise and fall times in an NLTL to this date. Neither are we aware of any demonstration of such NLTLs in silicon-based CMOS process technologies.

In this work, we first show a soliton line on a conventional silicon technology which can achieve very narrow pulses, with a bandwidth in excess of the cut-off frequency, $f_{T}$, of the fastest transistor in this process. Next, we demonstrate that using a favorable characteristic of MOS varactors leading to a different kind of non-linearity, we can improve both the rise and fall times, simultaneously. This is not possible with the nonlinear elements commonly used in NLTLs (e.g., reverse bias PN junctions). Neither can it be done using transistors, as they are limited by their $f_{T}$.

In this paper, the propagation of soliton waves in a non-linear transmission line will be studied. Then, we introduce two different types of non-linear transmission lines to generate narrow puises and to sharpen pulse transitions, respectively. Finally, we show the experimental results verifying the agreement between the theory and the measurement.
The Theory of Non-Linear Transmission Line

In this section, we review the basic theory behind non-linear transmission lines and their use for pulse narrowing and edge sharpening in subsections $A$ and $B$, respectively.

Fig. 1 shows an example of a non-linear transmission line using inductors, $l$, and voltage dependent (and bence non-linear) capacitors, $c(V)$.

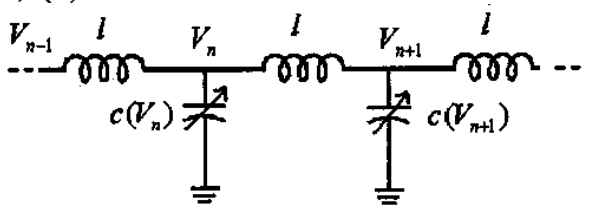

Figure 1. A non-linear transmission line

By applying KCL at node $n$, whose voltage with respect to ground is $V_{n}$, and applying $\mathrm{KVL}$ across the two inductors connected to this node, one can easily show the voltages of adjacent nodes on this NLTL are related via [5]:

$$
l \frac{d}{d t}\left[c\left(V_{n}\right) \frac{d V_{n}}{d t}\right]=\left(V_{n+1}+V_{n-1}-2 V_{n}\right)
$$

The right-hand side of (1) can be approximated with partial derivatives with respect to distance, $x$, from the beginning of the line, assuming that the spacing between two adjacent sections is $\delta$ (i.e., $x_{n}=n \delta$.) An approximate continuous partial differential equation can be obtained by using the Taylor expansions of $V(x-\delta), V(x)$, and $V(x+\delta)$ to evaluate the right hand side of (1). Assuming a small $\delta$, and ignoring the high order terms, we obtain:

$$
L \frac{\partial}{\partial t}\left[C(V) \frac{\partial V}{\partial t}\right]=\frac{\partial^{2} V}{\partial x^{2}}+\frac{\delta^{2}}{12} \frac{\partial^{4} V}{\partial x^{4}}
$$

where $C$ and $L$ are the capacitance and inductance per unit length, respectively. It is noteworthy that for a continuous transmission line $(\delta \rightarrow 0)$, (2) reduces to:

$$
L \frac{\partial}{\partial t}\left[C(V) \frac{\partial V}{\partial t}\right]=\frac{\partial^{2} V}{\partial x^{2}}
$$

A. Pulse Narrowing Non-Linear Transmission Lines

In this sub-section, we approximate the capacitor's voltage dependence using the following first-order linear relationship

$$
C(V)=C_{o}(1-b V)
$$

where $C_{0}$ and $b$ are constants. In this case, (2) reduces to

$$
\frac{\partial^{2} V}{\partial t^{2}}-\frac{1}{L C_{o}} \frac{\partial^{2} V}{\partial x^{2}}=\frac{\delta^{2}}{12} \frac{1}{L C_{o}} \frac{\partial^{4} V}{\partial x^{4}}+\frac{b}{2} \frac{\partial^{2}\left(V^{2}\right)}{\partial t^{2}}
$$

where the left-hand side is the classic wave equation and the terms on the right-hand side represent dispersion and non-linearity, respectively.

If the effect of the dispersive and non-linear terms in (5) are on the same order of magnitude it is possible to have a single pulse solution for (5) with a profile that does not change as it propagates with velocity, $v$. A propagating mode solution can be obtained by converting the partial differential equation (PDE) of (5) to an 
ordinary differential equation (ODE) by a simple change of variable: $u=x$-vt. This solution is:

$$
V(x, t)=\frac{3\left(v^{2}-v_{0}^{2}\right)}{b v^{2}} \operatorname{sech} 2\left[\frac{\sqrt{3\left(v^{2}-v_{0}^{2}\right)}}{v_{0}} \frac{(x-v t)}{\delta}\right]
$$

where $v$ is the propagation velocity of the pulse and $v_{0}=1 / \sqrt{L C_{0}}$.

This solution is shown in Fig. 2 for three different values of $L$ and $C$, and hence different $\delta$. Note that this solution is not a function of the input waveform, and thus any arbitrary input will eventually turns into (6) going through a line which is long enough, if it has enough energy.

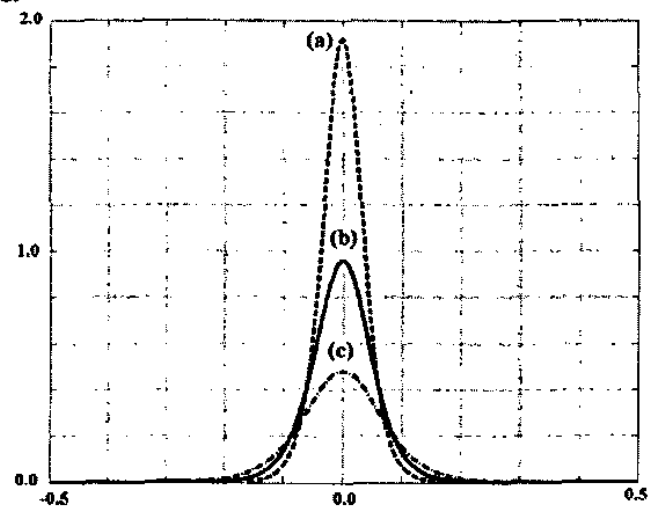

Figure 2. Three normalized soliton shapes for different values of $L$ and $C$ (a) $L=1 \mathrm{nH}$ and $C=1 \mathrm{nF}$ (b) $L=2 \mathrm{nH}$ and $C=2 \mathrm{nF}$ (c) $L=4 \mathrm{nH}$ and $C=4 \mathrm{nF}$

As can be seen from (6), the peak amplitude is a function of the velocity. Defining an effective capacitance, $C_{e f}$, so that $v=1 / \sqrt{L C_{e f f}}$, the pulse height is given by:

$$
v_{\max }=\frac{3}{b} \cdot \frac{v^{2}-v_{0}^{2}}{v^{2}}=\frac{3}{b}\left(1-\frac{C_{e f f}}{C_{0}}\right)
$$

Using (4), we can relate $C_{\text {eff }}$ to an effective voltage $V_{\text {eff }}$ It is easy to show that

$$
V_{e f f}=\frac{V_{\max }}{3}
$$

So it is the capacitance at one-third the peak amplitude that determines the effective propagation velocity. Using (6)-(8) we can easily calculate the half-height width of the pulse to be:

$$
W \approx \frac{\delta}{v} \frac{v_{0}}{\sqrt{\left(v^{2}-v_{0}^{2}\right)}}
$$

As can be seen, in a weakly dispersive and non-linear transmission line, the non-linearity can counteract the normally present dispersive properties of the line maintaining solitary waves that propagate without dispersion. This behavior can be explained using the following intuitive argument: The instantaneous propagation velocity at any given point in time and space is given by $1 / \sqrt{L C}$. In the presence of a non-linear capacitor with a characteristic given by (4), the instantaneous capacitance is smaller for higher voltages. Therefore, the points closer to the crest of the voltage waveform experience a faster propagation velocity and produce a shock-wave front due to the nonlinearity, as shown symbolically in the upper part of Fig. 3. Note that this is not a real waveform and more a fictitious representation of how each point on the curve tends to evolve. On the other hand, dispersion of the line causes the waveform to spread out, as shown in the lower half of Fig. 3. For a proper non-linearity determined by (5), these two effects can cancel each other out.

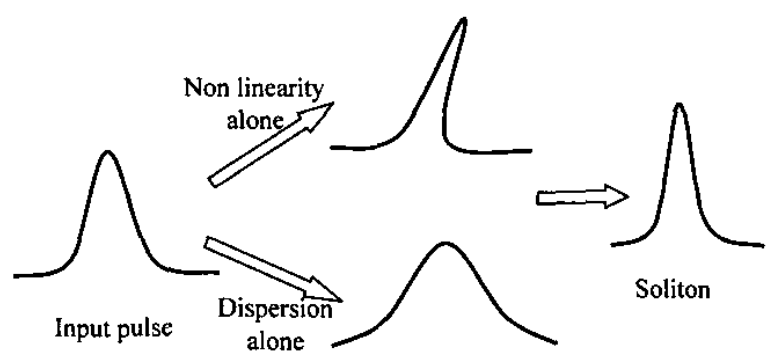

Figure 3. Dispersion and non-linear effects in the NLTL

A few important observations in this line are: 1) the velocity of the solitary wave increases with its amplitude, 2) pulse width decreases with increasing pulse velocity, 3) the width shrinks for higher amplitudes, 4) the sign of solution depends on sign of non-linearity factor, $b$; For a capacitor with a positive voltage dependence (e.g., an nMOS varactor in accumulation mode) we have

$$
C(V)=C_{o}(1+b V)
$$

resulting in upside down pulses.

Based on these results, to achieve large-amplitude narrow pulses, inductance and capacitance of the NLTL must be as small as possible and non-linearity factor, $b$, should be large enough to compensate the dispersion of the line.

It is also important to know the characteristic impedance of these lines (for impedance matching, etc.) As in a NLTL the capacitance is a function of voltage, we can only define an effective semi-empirical value for the characteristic impedance. Simulations results indicate that one can approximate $Z_{\text {eff }}$ using the capacitance at $V_{\text {eff }}$ defined in (8), i.e.,

$$
z_{\text {eff }}=\sqrt{\frac{L}{C\left(V_{\max } / 3\right)}}
$$

B. Edge Sharpening Lines

It is possible to design NLTLs to sharpen the pulse transitions. This is particularly useful for digital transmission (e.g., NRZ data). Unfortunately, all the efforts in the past [6] have resulted in sharpening of only one of the rising and falling edges. This, however, has very little practical value, as both transitions are equally important in common NRZ digital systems. This problem can be traced back to the monotonic dependence of the non-linear capacitive elements used in NLTL on the voltage (e.g., reverse biased PN junction, or the ideal behavior of (4) and (10)).

Fortunately, CMOS processes offer different characteristics for nonlinear capacitors that can be exploited to achieve simultaneous edge sharpening for both rising and falling edges. More specifically, accumulation mode MOS varactors [7] (an nMOS capacitor in an nwell) offer non-monotonic voltage dependence. Particularly, the secondary reduction of capacitarce shown in Fig. 4 due to polysilicon depletion $[8,9]$ and short-channel charge quantization [9] effects can be used for edge sharpening.

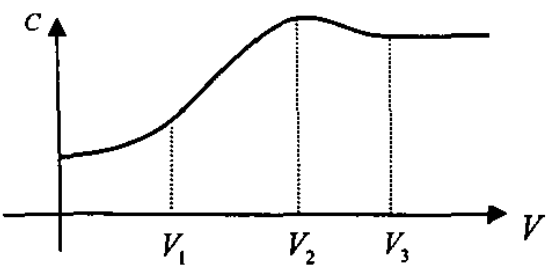

Figure 4. Capacitance versus voltage for a MOSVAR

Fig. 5 shows symbolically how one can use the behavior of Fig. 4 to sharpen both edges. First, let us focus on the rise-time reduction. 
Consider the rising edge shown in the upper part of Fig. 5 . Initially the voltage is low, which corresponds to a smaller capacitance per Fig. 4, and hence a faster instantaneous propagation velocity for the lower end of the pulse. As the voltage goes up, the capacitance increases, resulting in an increase in the instantaneous propagation velocity. This pushes the lower end of the transition forward in time and results in sharpening of the rising edge. This effect is symbolically shown in the fictitious middle waveform of Fig. 5 . The fall time reduction can be explained using the lower part of Fig. 5 . This is where the non-monotonic behavior of Fig. 4 plays its role. The upper part of the transition (voltages above $V_{2}$ ) will be accelerated due to the reduction of the capacitance and will create an advancing front as symbolically shown in the middle waveform of Fig. 5. The lower capacitance at the very low voltages can generate a leading tail, which will be partially dissipated by the line'.

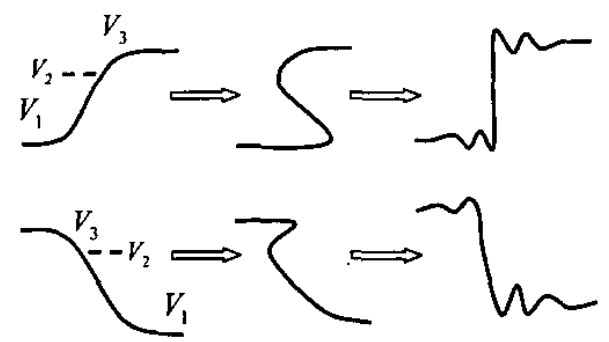

Figure 5. How rise and fall time vary within the NLTL.

While the above explanation based on a simplified memory-less description of the line provides a basic intuition for its operation, a complete description can only be obtained by solving the differential equation in (2) to account for the memory of the system. Our numerically solution of (2) also confirms that as long as the input voltage range exceeds voltages, $V_{l}$ and $V_{3}$, for a range of $L$ 's and $C$ 's, the line sharpens both rising and falling edges, simultaneously.

\section{Gradually Scaled NLTL}

One problem in pulse narrowing NLTLs is that if the input pulse is wider than a certain minimum related to the natural pulse width of the line in (9), it is incapable of concentrating all that energy into one pulse and instead the input pulse degenerates into multiple soliton pulses, as shown in the simulated upper waveforms of Fig.7. This is an undesirable effect that cannot be avoided in a standard line.

We have found a solution for this problem by using gradually scaled non-linear transmission lines. We notice that the characteristic pulse width of the line is controlled by the node spacing, $\delta$, and the propagation velocity, $v$, which is in tum controlled by, $L$ and $C$. Thus, we use a gradual line consisting of several sections that are gradually scaled to have smaller characteristic pulse width, as shown in Fig. 6.

The first few sections have the widest characteristic pulse, meaning that their output is wider and has smaller amplitude. As a result, the input pulse will cause just one pulse at the output of these sections. The following sections have a narrower response and the last section has the narrowest one. This will guarantee the gradual narrowing of the pulses and avoids degeneration. Each section has to be long enough so that the pulse can reach the section's steady-state response

' We hypothesize that other dynamic effects in the MOS varactor help edge sharpening, e.g., the processes of charge being attracted from the $\mathrm{n}^{+}$ diffusions to the channel and repelling them are not exact inverses of each other over short time intervals. Some of the repelled accumulation charges will be absorbed inside the well. This changes the response time of the capacitor and keeps it higher for a longer period of time for the falling edge. before entering next section.

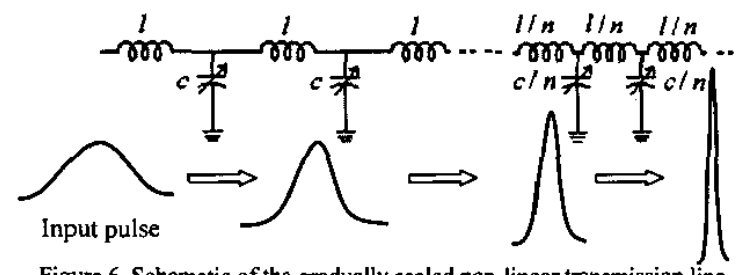

Figure 6. Schematic of the gradually scaled non-linear transmission line

The waveforms of this gradually scaled NLTL are shown in lower part of Fig. 7, demonstrating the effectiveness of this technique. It is noteworthy that this gradually scaling technique is also applicable to the edge sharpening lines and does improve their performance, too.
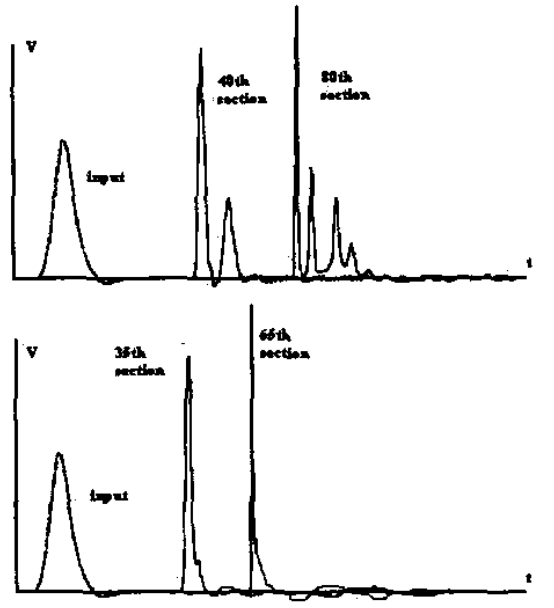

Figure 7. Output waveforms of the normal and gradual soliton line

Simulation

A. Pulse Narrowing Lines

We have designed a pulse narrowing NLTL using MOS varactors and metal micro-strip transmission lines in a $0.18 \mu \mathrm{m}$ BiCMOS process. We simulated the transmission lines in Sonnet and the complete NLTL in ADS. The output waveform of the line to a $65 \mathrm{ps}$ input pulse is shown in Fig. 8. This silicon-based NLTL produces pulses as narrow as $2.5 \mathrm{ps}$ (half amplitude width) with a $0.8 \mathrm{~V}$ amplitude. Active devices in this process are incapable of producing pulses nearly as narrow as these.

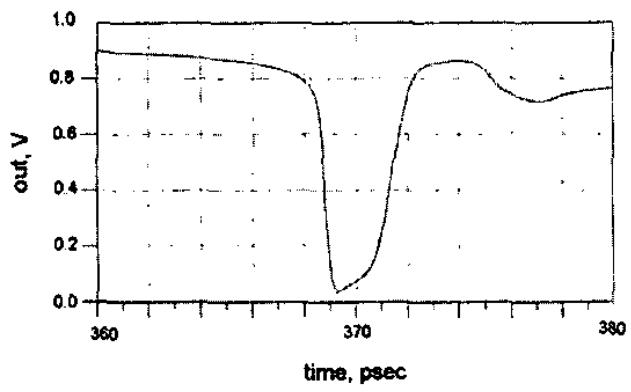

Figure 8. Simulated output waveform of the pulse narrowing line

\section{B. Edge Sharpening Lines}

We have also designed an edge sharpening non-linear transmission line using MOS varactors and gradual scaling of the lines in the same process. Fig. 9 shows the simulated input and the output waveforms of this line. 


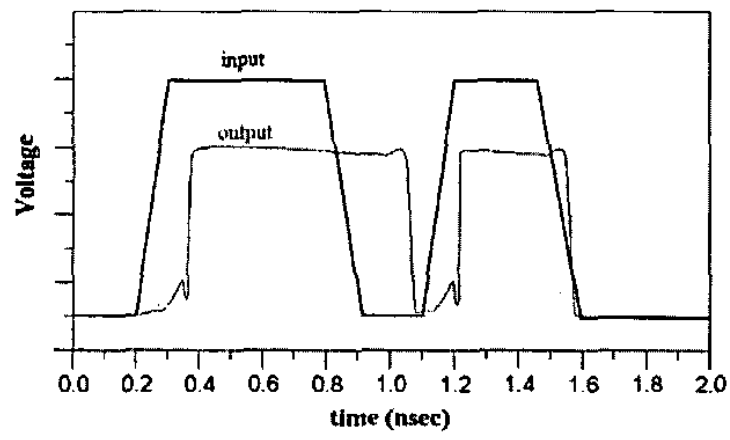

Figure 9. Simulated input and output waveforms of the edge sharpening line

The output pulses have a reduced rise and fall times of $1.5 \mathrm{ps}$ and $20 \mathrm{ps}$, respectively, because of the asymmetric behavior of non-linear element for two edges. We have also tested this line with a pseudorandom data source and verified its functionality for any arbitrary data sequence.

\section{Experimental Results}

Both lines were fabricated in a $0.18 \mu \mathrm{m}$ BiCMOS technology. We use RF probes to apply input to the line and to measure its output waveform. A $50 \mathrm{GHz}$ sampling oscilloscope is used to measure the input and output waveforms, as shown in Fig. 10 and Fig. 11.

A k-connector system of probes, connectors, and cables with a bandwidth of approximately $40 \mathrm{GHz}$ is used to bring the data to the oscilloscope. The main challenge in this measurement is the low bandwidth of the measurement system compared to the signal bandwidth. To the first order, this system can be approximated as a first-order system with a single pole at $40 \mathrm{GHz}$. It can be easily shown that the $10 \%$-to-90\% rise-time of such system is around $9 \mathrm{ps}$, which indicates that it is not possible to resolve rise times lower than 9 ps and pulse widths lower than 18 ps.

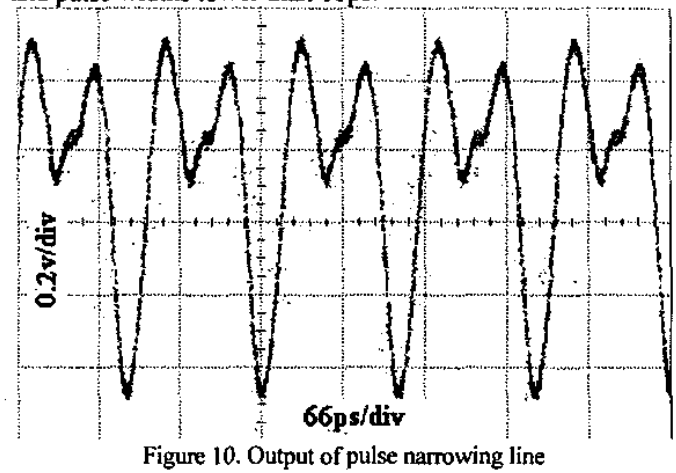

The rise time of two cascaded systems can be estimated using [10]:

$$
t_{\text {rise }}=\sqrt{t_{\text {risel }}^{2}+t_{\text {rise } 2}^{2}}
$$

Using this equation and the simulated rise and fall times, we should expect $9.1 \mathrm{ps}$ and $22 \mathrm{ps}$ at the output of a first-order system with a bandwidth of $40 \mathrm{GHz}$. Measurement waveforms of Fig. 11 show measured output rise and fall times of $11 \mathrm{ps}$ and $25 \mathrm{ps}$, respectively. These numbers are in agreement with the simulated results considering the additional bandwidth degradation due to the pads and the multi-pole nature of the system. The rise and fall times do not change with the input amplitude which verifies the non-linear behavior of the line.

\section{Conclusion}

We have analyzed pulse narrowing and edge sharpening passive' non-linear transmission lines, using MOS varactors and the novel gradual scaling lines, showing simultaneous edge sharpening for both rising and falling edges in a silicon substrate. The experimental results show considerable improvement in the rise and fall times of the pulses.

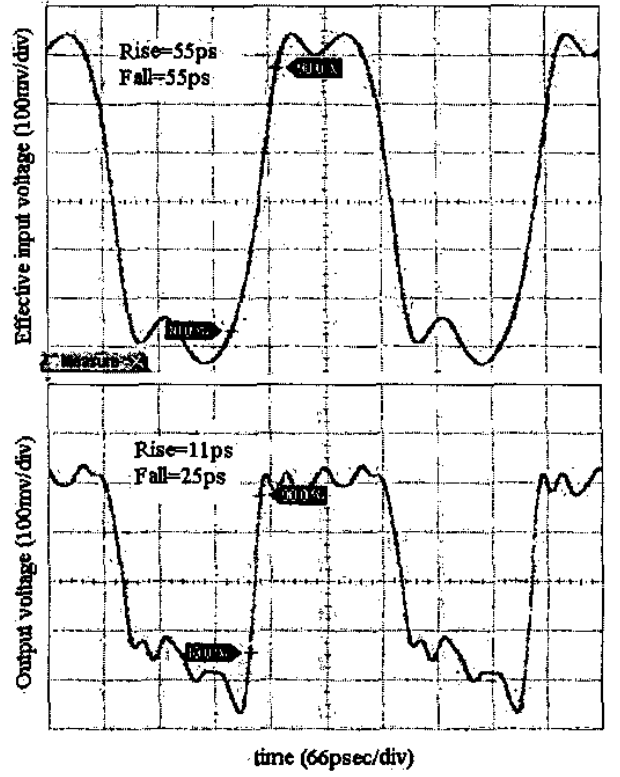

Figure 11. Input and output waveforms of the edge sharpening line

\section{Acknowledgements}

Authors would like to thank helpful discussions with D. Ham, H. Wu, A. Komijani, C. White, J. Buckwalter, M. Taghivand, H. Hashemi, S. Kee, B. Analui, and A. Natarajan of Caltech and Prof. M. Horowitz of Stanford University. They aiso acknowledge the generous support of Lee Center, IBM Corp., Agilent Tech., and NSF,

References

J. S. Russell, "Report on Waves," Report of the fourteenth meeting of the British Association for the Advancement of Science, pp. 31190, Plates XLVII-LVII, York, Sept. 1844 (London, 1845).

P. G. Drazin, and R. S. Johnson, Solitons, Cambridge University Press, Cambridge, 1989.

J. R. Tailor, Optical Solitons - Theory and Experiment, Cambridge University Press, Cambridge, 1992.

E. Infeld, and G. Rowlands, Nonlinear Woves, Solitons and Chaos, Cambridge University Press, Cambridge, 1990.

M. Remoissenet, Waves called Solitons: Concepts and Experiments, Springer-Verlag, Berlin, 1994.

M. G. Case, Nonlinear Transmission lines for Picosecond Pulse, Impulse and Millimeter-Wave Harmonic Generation, Ph.D. dissertation, University of California Santa Barbara, July 1993.

E. Kameda, T. Matsuda, Y. Emura, and T. Ohzone, "Study of the Current-Voltage Characteristics in MOS Capacitors with SiImplanted Gate Oxide," Solid-State Electronics, vol. 43, no. 3, pp. 555-63, March 1999.

[8] S. Matsumoto, K. Hisamitsu, M. Tanaka, H. Ueno, M. MiuraMattausch, Mattausch $\mathrm{H}$, et al. "Validity of Mobility Universality for Scaled Metal-Oxide-Semiconductor Field-Effect Transistors Down to $100 \mathrm{~nm}$ Gate Length," Journal of Applied Physics, vol. 92, no. 9, pp. 5228-32, Nov. 2002.

[9] L. Larcher, P. Pavan, F. Pellizzer, G. Ghidini, "A New Model of Gate Capacitance as a Simple Tool to Extract MOS Parameters," IEEE Transactions on Electron Devices, vol. 48, no. 5, pp. 935-45, May 2001.

[10] T. H. Lee, The Design of CMOS Radio-Frequency Integrated Circuits, Cambridge University Press, Cambridge, 1998. 